\title{
Parallel Implementation of Yee Algorithm 2D Using MPI
}

\author{
Adamu A. Isah, Awwal A. Babajo \\ Computer Science Department, Kaduna State University, Kaduna, Nigeria \\ Email: adamuaisah88@gmail.com
}

How to cite this paper: Isah, A.A. and Babajo, A.A. (2020) Parallel Implementation of Yee Algorithm 2D Using MPI. $A d$ vances in Internet of Things, 10, 11-15. https://doi.org/10.4236/ait.2020.102002

Received: January 26, 2020

Accepted: April 25, 2020

Published: April 28, 2020

Copyright $\odot 2020$ by author(s) and Scientific Research Publishing Inc. This work is licensed under the Creative Commons Attribution International License (CC BY 4.0).

http://creativecommons.org/licenses/by/4.0/

\begin{abstract}
The examination work clarifies a practical, superior registering stage for the parallel usage of the FDTD calculation on PC bunches utilizing the message-passing interface (MPI) library, which is a neighborhood framework comprising of various interconnected (PCs), and is now generally utilized for parallel figuring. In this paper, we describe the essential elements of a parallel algorithm for the FDTD method using the MPI (message passing interface). At present, the Internet of Things (IoT) has attracted more and more researchers' attention. Parallel FDTD method is applied to analyze the electromagnetic problems of the electrically large targets. This paper presents the concept of "the optimum virtual topology" for MPI based parallel FDTD. Parallel FDTD method is applied to analyze the electromagnetic problems of the electrically large targets on super computer.
\end{abstract}

\section{Keywords}

Distributed, Shared, Memory, Computer Architectures, Parallel Computing

\section{Introduction}

The finite-difference time-domain is a numerical investigation method utilized for displaying computational electrodynamics (finding inexact answers for the related arrangement of differential conditions). FDTD tackles the electromagnetic wave condition in the time area utilizing limited contrast approximations. Nowadays, the method is amongst the most mainstream method arrangement of electromagnetic equations and most differential problems [1] [2].

\section{Purpose of Study}

Performing errands on remote PCs for circulated occupations to exploit multi- 
core workstations (FDTD, varFDTD solvers) to beat the confinement size of numerous centers so as to have a usage with great productivity qualities and low execution time. To comprehend FDTD scientifically, just as some essential FDTD calculation, since it appears to have a high and proficient parallelization. To actualize parallelizing so as to diminish memory use and enhance the ideal opportunity for execution. The 6 fractional differential conditions of the FDTD numerical calculation.

\section{Application and Uses of FDTD}

- In electromagnetic reenactment in radar cross-segment counts

- Used in microwaves

- Antenna plan investigation

- Electromagnetic similarity examination example difference engines

- Medical research, for example, bosom malignancy location

- Computer chips and circuit plan example computer Architecture

- Physics-based flag handling and imaging example image processing

\section{Literature Review}

By and large Multiprocessors can be either: Shared memory, Distributed memory or Distributed-shared memory [3]. We have to parallelize FDTD to fulfill highlights like load balance, memory use balance, limit correspondence overhead, and diminish successive bottlenecks and versatility [4].

\section{Methodology}

Consequently, a substantial number of memory and computational time will be devoured when enormous measurement and complex structure object is considered, which is past the ability of single PC. The exploration calculation of this investigation depends on FDTD and MPI, utilizing C and its accuracy will be tried by contrasting diverse outcomes individually [5].

\section{Parallel Programming}

In straightforward terms it's a kind of calculation that permits different computations or execution of assignments at the same time [6]. Parallel program comprises of performs various tasks running on numerous processors holding on to be assembled or set of directions executed by a processor [7]. Taking current perceptions of climate and handling the information with PC models to gauge the future condition of climate. Used to think about abundance of sea utilizing multiprocessors having substantial computational power with low power prerequisites [8]. Parallel handling is utilized for demonstrating of economy of a country/world. High association between processors alongside Shared memory modules can impart computations to convey them crosswise over the greatest number of processors as you can inspire recreations to finish quicker, enhance exactness, or reenact greater physical frameworks [9]. 
They incorporate MPI which is clarified all through these bit of paper, VALU vector number-crunching rationale unit, AVX propelled vector expansions, CUDA figure brought together gadget curve, PVM parallel virtual machines and NUMA non-uniform memory get to. Utilized essentially for 2-D applications [10]. It is a solitary chip processor that makes lighting impacts and changes protests each time a 3D scene is redrawn. These are numerically escalated assignments, which generally, would put a significant strain on the CPU. Lifting this weight from the CPU opens up Cycles that can be utilized for different errands. CUDA is a parallel processing stage and programming model developed by NVIDIA [11]. It empowers emotional increments in registering execution by saddling the intensity of the illustrations handling unit (GPU) [12]. Is a product bundle that licenses heterogeneous gathering of UNIX as well as Windows PCs snared together by a system to be utilized as a solitary substantial parallel PC [13].

\section{Discussion of Results and Comparisons}

The investigation utilizes MPI to build up a broadly utilized standard for composing message passing projects [2]. The interface sets up a down to earth, versatile, proficient and adaptable standard, indicates a library in a dialect in-subordinate shape and gives a predicament among $\mathrm{C}$ and FORTRAN. It has increased wide acknowledgment in parallel registering extending from Massively Parallel frameworks to network of PCs and workstations [14] [15].

\section{Summary}

The proposed framework relies upon logically applying forward and differencing in time for the conduction of execution and aggregations, there is increasingly compelling for generous Courant numbers [8]. This work introduces another structure that permits less machine memory stockpiling, and all the more essentially CPU time, for example in the computational region is a lot littler than the wavelength of the strategies [7]. Numerical recreation perceptions exhibits that the new procedure is amazingly capable, and the outcomes are extraordinarily well with FDTD strategy as clarified more in the table [11].

\section{Recommendations}

The (FDTD) framework for settling the full-wave Maxwell's correlations has been starting late extended to give correct and numerically consistent activity for time steps outperforming each other quite far. One such class of issues is the examination of quick execution time which interconnects the strategies that are as often as possible required for the correct assurance of a commonplace electromagnetic wave marvels.

\section{Future Work}

To simplify and accelerate the algorithm, an MPI approach is used. The inter-process communications are optimized by the use of derived data types. A 
general approach is also explained for parallelizing the auxiliary tools, such as far-field computation, thin-wire treatment, etc. [15]. For some $\mathrm{X}$ and $\mathrm{Y}$ axis like in the figures, we have used a new method that makes it unnecessary to split the field components. This considerably simplifies the computer programming, and is compatible with the parallel algorithm [15].

\section{Conclusion}

It has been seen that the parallel usage of the proposed calculation gives a critical decrease in reproduction time expected to refresh field segments just as the correspondence time expected to play out the interprocessor correspondence, when parceling the computational area over numerous processors, particularly for little spaces, the proficiency of the parallelization will achieve a confinement because of increment in the correspondence time between the processors.

\section{Acknowledgements}

We would like to express special thanks of gratitude to our supervisors Dr. Muhammad Aminu as well as our parents (Alhaji Abubakar and Alhaji Awwal) who gave us the golden opportunity to do this wonderful project on this interesting topic which has also helped us in doing a lot of Research and we came to know about so many new things we are really thankful to them.

Secondly, I would also like to thank my friends who helped us a lot in finalizing this project within the limited time frame.

To our well wishers lastly, we thank you all and the almighty may guide us all the time.

\section{Conflicts of Interest}

The authors declare no conflicts of interest regarding the publication of this paper.

\section{References}

[1] Ramadan, O., Akaydin, O., Salamah, M. and Oztoprak, A.Y. (2004) Parallel Implementation of the Wave-Equation Finite-Difference Time-Domain Method Using the Message Passing Interface. In: International Symposium on Computer and Information Sciences, Lecture Notes on Computer Science (LNCS), Vol. 3280, Springer, Berlin, 810-818. https://doi.org/10.1007/978-3-540-30182-0_81

[2] Yee, K.S. (1966) Numerical Solution of Initial Boundary Value Problems Involving Maxwell's Equations in Isotropic Media. IEEE Transaction on Antennas and Propagation, 14, 302-307. https://doi.org/10.1109/TAP.1966.1138693

[3] Kusaf, M. and Oztoprak, A.Y. (2009) Multi Split-Step Unconditionally Stable Finite Difference Time Domain Methods. Microwave and Optical Technology Letters, 51, 2646-2649. https://doi.org/10.1002/mop.24704

[4] Capoglu, I.R., Taflove, A. and Backman, V. (2012) A Frequency-Domain Near-Field-toFar-Field Transform for Planar Layered Media. IEEE Transactions on Antennas and Propagation, 60, 1878-1885. https://doi.org/10.1109/TAP.2012.2186253 
[5] Avinash Reddy, M., et al. (2014) Gain and Bandwidth Enhancement of a MSP Antenna over Multiple-Layer Dielectric Substrate. International Journal of Advances in Computer, 3, 55-57.

[6] Yu, W., Yang, X., Liu, Y., Mittra, R. and Muto, A. (2011) Advanced FDTD Method: Acceleration, Parallelization and Engineering Applications. Artech House, Norwood.

[7] Yee, K.S., Chen, J.S. and Chang, A.H. (1992) Conformal Finite Difference Time Domain (FDTD) with Overlapping Grids. IEEE Antennas.

[8] Taflove, A. and Hagness, S. (2005) Computational Electrodynamics the Finite-Difference Time Domain Method. Artech House, Norwood. https://doi.org/10.1002/0471654507.eme123

[9] Catarinucci, L., Guglielmi, S., Colella, R. and Tarricone, L. (2014) Compact Switched-Beam Antennas Enabling Novel Power-Efficient Wireless Sensor Networks. IEEE Sensors Journal, 14, 3252-3259. https://doi.org/10.1109/JSEN.2014.2326971

[10] Zheng, F. and Chen, Z. (2001) Numerical Dispersion Analysis of the Unconditionally Stable 2-D ADI-FDTD Method. IEEE Transactions on Microwave Theory and Technique, 49, 1006-1009. https://doi.org/10.1109/22.920165

[11] Hastings, F.D., et al. (1996) application of the Perfectly Matched Layer (PML) Absorbing Boundary-Condition to Elastic-Wave Propagation. The Journal of the Acoustical Society of America, 100, 3061-3069. https://doi.org/10.1121/1.417118

[12] Fu, W. and Tan, E. (2004) Development of Split-Step FDTD Method with Higher-Order Spatial Accuracy. IEEE Electronics Letter, 40, 1252-1253. https://doi.org/10.1049/el:20046040

[13] Zhang, F., Chen, Z. and Zhang, J. (2000) Towards the Development of a Three Dimensional Unconditionally Stable Finite Difference Time Domain Method. IEEE Transactions on Microwave Theory and Techniques, 48, 1550-1558. https://doi.org/10.1109/22.869007

[14] Ruhl, H., Moschuring, N. and Elkina, N. (2012) Computational Physics Course 17104 Lecture 9.

[15] Jaakko, S. and Theodoros, D. (2000) Reduction of Numerical Dispersion in FDTD Method through Artificial Anisotropy. IEEE Transactions on Microwave Theory and Techniques, 48, 582-588. https://doi.org/10.1109/22.842030 